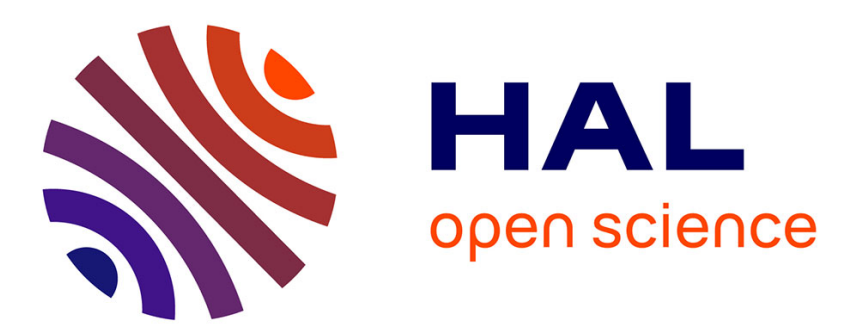

\title{
Nonlinear kinematics Reissner's beam with combined hardening/softening elastoplasticity
}

\author{
Ismar Imamovic, Adnan Ibrahimbegovic, Esad Mesic
}

\section{To cite this version:}

Ismar Imamovic, Adnan Ibrahimbegovic, Esad Mesic. Nonlinear kinematics Reissner's beam with combined hardening/softening elastoplasticity. Computers \& Structures, 2017, 189, pp.12-20. 10.1016/j.compstruc.2017.04.011 . hal-01997365

\section{HAL Id: hal-01997365 \\ https://hal.utc.fr/hal-01997365}

Submitted on 5 Feb 2019

HAL is a multi-disciplinary open access archive for the deposit and dissemination of scientific research documents, whether they are published or not. The documents may come from teaching and research institutions in France or abroad, or from public or private research centers.
L'archive ouverte pluridisciplinaire HAL, est destinée au dépôt et à la diffusion de documents scientifiques de niveau recherche, publiés ou non, émanant des établissements d'enseignement et de recherche français ou étrangers, des laboratoires publics ou privés. 


\title{
Nonlinear kinematics Reissner's beam with combined hardening/softening elastoplasticity
}

\author{
Ismar Imamovic $^{\mathrm{a}, \mathrm{b}}$, Adnan Ibrahimbegovic ${ }^{\mathrm{a}, *}$, Esad Mesic ${ }^{\mathrm{b}}$ \\ ${ }^{a}$ Laboratoire Roberval, Université de Technologie de Compiègne/Sorbonne Universités, France \\ ${ }^{\mathrm{b}}$ Faculty of Civil Engineering, University Sarajevo, Sarajevo, Bosnia and Herzegovina
}

\section{A R T I C L E I N F O}

\section{Article history:}

Received 9 January 2017

Accepted 19 April 2017

\section{Keywords:}

Reissner's beam

Geometrically exact beam

Hardening/softening response

\begin{abstract}
A B S T R A C T
In this work, we present geometrically non-linear beam finite element with embedded discontinuity which can represent elastoplastic constitutive behavior with both hardening and softening response. The constitutive equations are presented in rate form by using the multiplicative decomposition of deformation gradient. Formulation of elastoplastic response is presented in terms of stress resultants including the interaction between axial force, shear force and bending moment appropriate for metallic materials. The softening response is used to model the failure in connections, introducing displacement field discontinuity or a rotational hinge. The hinges or displacement discontinuity are presented in the framework of incompatible modes that can handle three different failure modes dealing with bending, shearing or axial deformation. With several numerical simulations, the FEM implementation is proven very robust for solving the problems of practical interest, such as push-over analysis.
\end{abstract}

(c) 2017 Elsevier Ltd. All rights reserved.

\section{Introduction}

The model capable of predicting the complete failure (collapse) of a frame structure is very important in the limit load design. Typical application is push-over analysis used in earthquake engineering; a nonlinear static analysis of a building structure subjected to an equivalent static load that is pushing a structure towards the limit capacity. This type of the analysis was developed in work [1] as incompatible modes in the small displacement framework. During push-over analysis of a structure, there are hinges that develop, in a step-by-step manner, leading to the failing mechanism. In structural analysis those hinges can be included by using static condensation method [7]. The incompatible mode method is more robust, while the static condensation method is more efficient. For improved prediction, it is necessary [1] to include geometric nonlinearities of the second order, indicating the need for improvement.

The truly large kinematics of steel frame structures combined with elastoplastic hardening/softening is the main novelty of this work. The ductile material like steel can handle large displacements and deformation of a structure during the limit load analysis. The geometrically exact beam with nonlinear kinematics and nonlinear constitutive behavior should be capable of following

\footnotetext{
* Corresponding author.

E-mail address: adnan.ibrahimbegovic@utc.fr (A. Ibrahimbegovic).
}

response of a structure to the complete failure (collapse). In this work, we propose elastoplastic beam element in geometrically nonlinear regime [4] that can handle softening response, which is included in the framework of incompatible modes.

In the formulation of the proposed beam element we use, as the starting point, the previous works $[4,11]$. The proposed beam element includes nonlinear kinematics and nonlinear constitutive response. The constitutive behavior is defined as plasticity with linear hardening that includes interaction between axial force, shear force and bending moment. The evolution equations for internal variables are developed in rate form, imposing the need to employ a numerical time integration scheme, -here chosen as the backward Euler scheme.

The main novelty concerns the beam model's ability to reach the ultimate capacity of a cross section, activating one of three failure modes, which represent non-linear softening response in either bending moment, shear or axial force. These failure modes are handled by field discontinuity as incompatible modes, see [5]. In this work, we presume that only one softening failure mechanism can be activated at the time. The outline of the paper is as follows.

In the next section, we present the main ingredients of the geometrically exact beam with the elastoplastic constitutive response. The interaction between axial force, shear force and bending moment is taken in the elastoplastic regime, while the axial response remains elastic. Section 2 presents corresponding kinematic enhancement in terms of "discontinuity" or "jump" in 
the displacement field or the rotational field depending upon the activated failure mode. The enhancement is included as an incompatible mode in the geometrically nonlinear framework. Section 3 deals the FEM implementation, while Section 4 presents the results of several numerical simulations. Section 5 contains the conclusions.

\section{Reissner's beam with non-linear kinematics}

In this section, we give a detailed formulation of the twodimensional beam in the framework of large displacement and large elastoplastic strains. The formulation of Reissner's beam [10] kinematics equations employs rotated strain measure. The linearization of these strain measures reduce the strains of the Timoshenko beam $[4,9]$. The plastic strains corresponding to stress resultant follow from yield criterion introducing the interaction between axial force, shear force and bending moment. The equations are expressed in rate form [11]. The consistent linearization of the weak form of equilibrium equations provides tangent stiffness matrix, for both material and geometric part.

Providing the beam element with the embedded discontinuity within the framework of a large displacement is needed for modeling softening phase. The later can concern the failure process in the connections, modeling separately the failure in bending, in shearing or in axial force. The multiplicative decomposition of the deformation gradient into regular and irregular parts corresponds to the additive decomposition of the rotated strain measure proposed by Reissner [10]. Moreover, the weak form of equilibrium equation has to be recast within the framework of incompatible modes [5], which allows handling of the embedded discontinuity calculation at the element level.

\subsection{Geometrically nonlinear kinematics}

In the framework of large displacement gradient theory, the position vector in deformed configuration can be written as

$\boldsymbol{\varphi}:=\boldsymbol{\varphi}_{\mathbf{0}}+\zeta \mathbf{t}=\left(\begin{array}{l}x+u \\ y+v\end{array}\right)+\zeta\left(\begin{array}{c}-\sin \psi \\ \cos \psi\end{array}\right)$

where $x$ and $y$ are coordinates in the reference configuration, $u$ and $v$ are displacement components in the global coordinate system, $\zeta$ is the coordinate along the normal to the beam axis in the reference configuration and $\psi$ is the rotation. The corresponding form of the deformation gradient $\mathbf{F}$ can be split into displacement part $\mathbf{F}_{u, v}$ and rotation part $\mathbf{F}_{\psi}$ as:

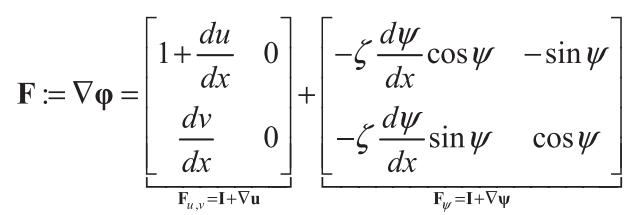

The failure mode in connection can be represented by jump in displacement components $u, v$ and in the rotation $\psi$, with the corresponding kinematic enhancement in terms of the "discontinuity". In the finite deformation framework, such a displacement discontinuity has to be introduced in deformed configuration [4]. This splits displacement field into the regular part $(\tilde{\bullet})$ and the "enhanced" part $(\overline{\bar{\bullet}})$ representing the corresponding displacement or rotation "jump". By introducing $\delta_{\bar{x}}$ as the Dirac function where the jump occurs, the additive decomposition of displacements and rotation gradient fields can be written as: $u(x, t)=\tilde{\tilde{u}}(x, t)+\left(N_{a}(x)+H(x)\right) \overline{\bar{u}}(t) \rightarrow \frac{\partial u}{\partial x}=\frac{\partial \tilde{\bar{u}}}{\partial x}+G_{a}(x) \overline{\bar{u}}+\delta_{\bar{x}} \overline{\bar{u}}=\frac{\partial \bar{u}}{\partial x}+\delta_{\bar{x}} \overline{\bar{u}}$

$v(x, t)=\tilde{\bar{v}}(x, t)+\left(N_{a}(x)+H(x)\right) \overline{\bar{v}}(t) \rightarrow \frac{\partial v}{\partial x}=\frac{\partial \tilde{\bar{v}}}{\partial x}+G_{a}(x) \overline{\bar{v}}+\delta_{\bar{x}} \overline{\bar{v}}=\frac{\partial \bar{v}}{\partial x}+\delta_{\bar{x}} \overline{\bar{v}}$

$\psi(x, t)=\tilde{\bar{\psi}}(x, t)+\left(N_{a}(x)+H(x)\right) \overline{\bar{\psi}}(t) \rightarrow \frac{\partial \psi}{\partial x}=\frac{\partial \tilde{\bar{\psi}}}{\partial x}+G_{a}(x) \overline{\bar{\psi}}+\delta_{\bar{x}} \overline{\bar{\psi}}=\frac{\partial \bar{\psi}}{\partial x}+\delta_{\bar{x}} \overline{\bar{\psi}}$

where $N_{a}(x)$ is interpolation function, $H(x)$ is Heaviside function and $G_{a}(x)$ is the first derivative of the interpolation function $N_{a}(x)$. By using last result (3) we can write the deformation gradient for both the displacement and the rotation fields, in terms of the multiplicative decomposition of:

$$
\begin{aligned}
\mathbf{F} & =\mathbf{I}+\nabla \overline{\mathbf{u}}+\delta_{\bar{x}} \nabla \overline{\overline{\mathbf{u}}}+\mathbf{I}+\nabla \bar{\psi}+\delta_{\bar{x}} \nabla \overline{\bar{\psi}} \\
& =(\mathbf{I}+\nabla \overline{\mathbf{u}})\left(\mathbf{I}+\delta_{\bar{x}} \frac{\nabla \overline{\overline{\mathbf{u}}}}{I+\nabla \overline{\mathbf{u}}}\right)+(\mathbf{I}+\nabla \bar{\psi})\left(\mathbf{I}+\delta_{\bar{x}} \frac{\nabla \overline{\bar{\psi}}}{I+\nabla \bar{\psi}}\right) \\
& =\overline{\mathbf{F}}_{\mathbf{u}, \mathbf{v}} \overline{\overline{\mathbf{F}}}_{\mathbf{u}, \mathbf{v}}+\overline{\mathbf{F}}_{\psi} \overline{\overline{\mathbf{F}}}_{\psi}
\end{aligned}
$$

From the polar decomposition of the deformation gradient $\mathbf{F}$, into rotation $\mathbf{R}$ and stretch $\mathbf{U}$, we define the rotated strain measure H:

$\mathbf{F}=\mathbf{R} \mathbf{U} \rightarrow \mathbf{U}=\mathbf{R}^{T} \mathbf{F}, \mathbf{R}=\left[\begin{array}{cc}\cos \psi & -\sin \psi \\ \sin \psi & \cos \psi\end{array}\right] \rightarrow \mathbf{H}=\mathbf{U}-\mathbf{I}$

where I is identity tensor. With the results (4) and (5), we can obtain the corresponding additive decomposition of the stretch tensor:

$$
\mathbf{U}=\mathbf{R}^{\mathrm{T}}\left(\mathbf{I}+\nabla \overline{\mathbf{u}}+\delta_{\bar{x}} \nabla \overline{\overline{\mathbf{u}}}\right)+\mathbf{R}^{\mathrm{T}}\left(\mathbf{I}+\nabla \bar{\psi}+\delta_{\bar{x}} \nabla \overline{\bar{\psi}}\right)=\underbrace{\overline{\mathbf{U}}^{u, v}+\delta_{\bar{x}} \overline{\overline{\mathbf{U}}}^{u, v}}_{\mathbf{U}^{u, v}}+\underbrace{\overline{\mathbf{U}}^{\psi}+\delta_{\overline{\overline{\mathbf{U}}}}}_{\mathbf{U}^{\psi}} \overline{\overline{\mathbf{U}}}^{\psi}
$$

where

$\overline{\mathbf{U}}^{\mathbf{u}, \mathbf{v}}=\left[\begin{array}{cc}\left(1+\frac{\partial \bar{u}}{\partial x}\right) \cos \psi+\frac{\partial \bar{v}}{\partial x} \sin \psi & 0 \\ -\left(1+\frac{\partial \bar{u}}{\partial x}\right) \sin \psi+\frac{\partial \bar{v}}{\partial x} \cos \psi & 1\end{array}\right] ; \overline{\overline{\mathbf{U}}}^{\mathbf{u}, \mathbf{v}}=\left[\begin{array}{cc}\overline{\bar{u}} \cos \psi+\overline{\bar{v}} \sin \psi & 0 \\ -\overline{\bar{u}} \sin \psi+\overline{\bar{v}} \cos \psi & 0\end{array}\right] \delta_{\overline{\mathbf{x}}}$

$\overline{\mathbf{U}}^{\psi}=\left[\begin{array}{cc}-\zeta \frac{\partial \bar{\psi}}{\partial x} & 0 \\ 0 & 1\end{array}\right] ; \overline{\mathbf{U}}^{\mathbf{u}, \mathbf{v}}=\left[\begin{array}{cc}-\zeta \overline{\bar{\psi}} & 0 \\ 0 & 0\end{array}\right] \delta_{\overline{\mathbf{x}}}$

Finally, we can write the internal virtual work in an alternative form that is more in line with the corresponding 3D representations [4]

$\int_{L} \int_{A} \hat{\mathbf{F}} \cdot \mathbf{P} d A d x=\int_{L} \int_{A} \hat{\mathbf{H}} \cdot \mathbf{T} d A d x$

where $\hat{\mathbf{F}}$ is variation of the deformation gradient, $\mathbf{P}$ is first PiolaKirchhoff stress. In last Eq. (7), we used the following result for Biot stress tensor $\mathbf{T}$ and corresponding rotated strain measures $\mathbf{H}$ and their variations $\hat{\mathbf{H}}$ :

$\mathbf{T}=\mathbf{R}^{\mathbf{T}} \mathbf{P} \rightarrow\left(\begin{array}{l}T^{11} \\ T^{21}\end{array}\right)=\mathbf{R}^{\mathbf{T}}\left(\begin{array}{l}P^{11} \\ P^{21}\end{array}\right)$

\subsection{Constitutive model and its rate form}

In the elastic regime the simplest set of constitutive equations for finite strain beam is chosen in terms of Biot stress resultants and rotated strain measure:

$\mathbf{T}=\mathbf{C}^{e} \mathbf{H}$ 
where $\mathbf{C}^{e}$ is the elastic modulus. In the plastic regime, we can split displacement and rotation gradients into elastic part $\left(\bullet^{e}\right)$ and plastic part $\left(\bullet^{p}\right)$ :

$$
\begin{aligned}
\overline{\mathbf{F}} & =\mathbf{I}+\nabla \overline{\mathbf{u}}^{\mathbf{e}}+\nabla \overline{\mathbf{u}}^{\mathbf{p}}+\mathbf{I}+\nabla \bar{\psi}^{\mathbf{e}}+\nabla \bar{\psi} \mathbf{p} \\
& =\left(\mathbf{I}+\nabla \overline{\mathbf{u}^{\mathbf{e}}}\right)\left(\mathbf{I}+\frac{\nabla \overline{\mathbf{u}}^{\mathbf{p}}}{\mathbf{I}+\nabla \overline{\mathbf{u}}^{\mathbf{e}}}\right)+(\mathbf{I}+\nabla \bar{\psi} \mathbf{e})\left(\mathbf{I}+\frac{\nabla \bar{\psi} \mathbf{p}}{\mathbf{I}+\nabla \bar{\psi} \mathbf{e}}\right) \\
& =\overline{\mathbf{F}}_{\mathbf{u}, \mathbf{v}}^{\mathbf{e}} \overline{\mathbf{F}}_{\mathbf{u}, \mathbf{v}}^{\mathbf{p}}+\overline{\mathbf{F}}_{\psi}^{\mathbf{e}} \overline{\mathbf{F}}_{\psi}^{\mathbf{p}}
\end{aligned}
$$

Multiplicative decomposition of the deformation gradient corresponds to the additive decomposition of the stretch tensor $\mathbf{U}$ :

$$
\mathbf{U}=\mathbf{R}^{\mathrm{T}}\left(\mathbf{I}+\nabla \overline{\mathbf{u}}^{e}+\nabla \overline{\mathbf{u}}^{p}\right)+\mathbf{R}^{\mathrm{T}}\left(\mathbf{I}+\nabla \bar{\psi}^{e}+\nabla \bar{\psi}^{p}\right)=\underbrace{\overline{\mathbf{U}}_{u, v}^{e}+\overline{\mathbf{U}}_{u, v}^{p}}_{\overline{\mathbf{U}}_{u, v}}+\underbrace{\overline{\mathbf{U}}_{\psi}^{e}+\overline{\mathbf{U}}_{\psi}^{p}}_{\overline{\mathbf{U}}_{\psi}}
$$

where

$$
\begin{aligned}
& \overline{\mathbf{U}}_{\mathbf{u}, \mathbf{v}}^{\mathbf{e}}=\left[\begin{array}{cc}
\left(1+\frac{\partial \bar{u}^{e}}{\partial x}\right) \cos \psi+\frac{\partial \bar{v}^{e}}{\partial x} \sin \psi & 0 \\
-\left(1+\frac{\partial \bar{u}^{e}}{\partial x}\right) \sin \psi+\frac{\partial \bar{v}^{e}}{\partial x} \cos \psi & 1
\end{array}\right] ; \\
& \overline{\mathbf{U}}_{\mathbf{u}, \mathbf{v}}^{\mathbf{p}}=\left[\begin{array}{cc}
\frac{\partial \bar{u}^{p}}{\partial x} \cos \psi+\frac{\partial \bar{\nu}^{p}}{\partial x} \sin \psi & 0 \\
-\frac{\partial \bar{u}^{p}}{\partial x} \sin \psi+\frac{\partial \bar{v}^{p}}{\partial x} \cos \psi & 0
\end{array}\right] \\
& \overline{\mathbf{U}}_{\psi}^{\mathbf{e}}=\left[\begin{array}{cc}
-\zeta \frac{\partial \bar{\psi}^{e}}{\partial x} & 0 \\
0 & 1
\end{array}\right] ; \overline{\mathbf{U}}_{\psi}^{\mathbf{p}}=\left[\begin{array}{cc}
-\zeta \frac{\partial \bar{\psi}^{p}}{\partial x} & 0 \\
0 & 0
\end{array}\right]
\end{aligned}
$$

The Helmholtz free energy can be defined as a quadratic form:

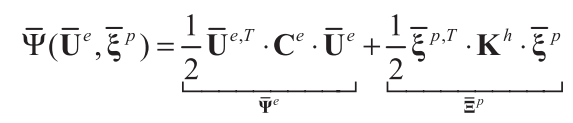

where $\overline{\mathbf{U}}^{\mathbf{e}}$ is elastic part of the stretch tensor, $\bar{\xi}^{p}$ is vector of hardening variables and $\mathbf{K}^{h}$ are corresponding hardening moduli. The yield criterion condition has to be satisfied:

$\bar{\phi}(\mathbf{T}, \overline{\mathbf{q}}) \leqslant 0$

where $\overline{\mathbf{q}}$ is vector of internal hardening stress like variables. The second principle of thermodynamics states that the plastic dissipation must remain non-negative:

$0 \leqslant \overline{\mathscr{D}}=\underbrace{\left(\mathbf{T}-\frac{d \bar{\Psi}^{e}}{d \overline{\mathbf{U}}^{\mathbf{e}}}\right) \dot{\mathbf{U}}^{e}}_{\overline{\mathscr{P}}^{e}=0}+\underbrace{\mathbf{T}_{\mathbf{U}^{p}}-\frac{\partial \bar{\Xi}^{p}}{\partial \bar{\xi}^{p}} \frac{d \bar{\xi}^{p}}{d t}}_{\overline{\mathscr{P}}^{p}}$

The principal of maximum plastic dissipation can be formulated [2] as the constrained minimization, where constraint is yield function (13). This can further be recast as corresponding unconstrained minimization by using Lagrange multiplier method:

$\min _{\mathbf{T}, \mathbf{q}} \max _{\dot{\gamma}}\left[\bar{L}^{p}(\mathbf{T}, \overline{\mathbf{q}}, \dot{\bar{\gamma}})=-\overline{\mathscr{D}}^{p}(\mathbf{T}, \overline{\mathbf{q}})+\dot{\bar{\gamma}} \cdot \bar{\phi}(\mathbf{T}, \overline{\mathbf{q}})\right]$

where $\dot{\bar{\gamma}}$ is the Lagrange multiplier. Regarding the Kuhn-Tucker optimality conditions, the result can be used to provide the evolution equations for internal variables in rate form along with the loading/unloading conditions:

$$
\begin{aligned}
& \frac{\partial \bar{L}^{p}}{\partial \mathbf{T}}=-\dot{\overline{\mathbf{U}}}^{p}+\dot{\bar{\gamma}} \frac{\partial \bar{\phi}}{\partial \mathbf{T}}=0 \Rightarrow \dot{\overline{\mathbf{U}}}^{p}=\dot{\bar{\gamma}} \frac{\partial \bar{\phi}}{\partial \mathbf{T}} \\
& \frac{\partial \bar{L}^{p}}{\partial \overline{\mathbf{q}}}=-\frac{\partial \bar{\xi}^{p}}{\partial t}+\dot{\bar{\gamma}} \frac{\partial \bar{\phi}}{\partial \overline{\mathbf{q}}}=0 \Rightarrow \frac{\partial \bar{\xi}^{p}}{\partial t}=\dot{\bar{\gamma}} \frac{\partial \bar{\phi}}{\partial \overline{\mathbf{q}}} \\
& \dot{\bar{\gamma}} \geqslant 0, \bar{\phi} \leqslant 0, \dot{\bar{\gamma}} \bar{\phi}=0
\end{aligned}
$$

The appropriate value of plastic multiplier $\dot{\bar{\gamma}}$ can be determined from the plastic consistency condition for the case of sustained plastic flow:

$\dot{\bar{\phi}}=0 \Rightarrow \dot{\bar{\gamma}}=\frac{\frac{\partial \bar{\phi}}{\partial \mathbf{T}} \mathbf{C}^{e} \dot{\overline{\mathbf{U}}}}{\frac{\partial \bar{\phi}}{\partial \mathbf{T}} \mathbf{C}^{e} \frac{\partial \bar{\phi}}{\partial \mathbf{T}}+\frac{\partial \bar{\phi}}{\partial \overline{\mathbf{q}}} \mathbf{K}^{h} \frac{\partial \bar{\phi}}{\partial \overline{\mathbf{q}}}}$

By replacing the last result in stress rate equation, we can obtain the elastoplastic modulus $\mathbf{C}^{e p}$ that should replace the elastic modulus $\mathbf{C}^{e}$ in plastic regime:

$C^{e p}=\mathbf{C}^{e}-\frac{\mathbf{C}^{e} \frac{\partial \bar{\phi}}{\partial \mathbf{T}} \otimes \mathbf{C}^{e} \frac{\partial \bar{\phi}}{\partial \mathbf{T}}}{\frac{\partial \bar{\phi}}{\partial \mathbf{T}} \mathbf{C}^{e} \frac{\partial \bar{\phi}}{\partial \mathbf{T}}+\frac{\partial \bar{\phi}}{\partial \overline{\mathbf{q}}^{p}} \mathbf{K}^{h} \frac{\partial \bar{\phi}}{\partial \overline{\mathbf{q}}^{p}}}$

We note in passing that the elastoplastic tangent stiffness above remains the same in the discrete problem, obtained by using the backward Euler time integration scheme.

In the softening regime the Helmholtz free energy can be written as a quadratic form in softening variables:

$\overline{\bar{\Psi}}\left(\overline{\bar{\xi}}^{s}\right)=\underbrace{\frac{1}{2} \overline{\bar{\xi}}^{s} \mathbf{K}_{s} \overline{\bar{\xi}} ;}_{\bar{\Xi}^{s}} ; \Psi(\cdot)=\bar{\Psi}(\cdot)+\overline{\bar{\Psi}}\left(\overline{\bar{\xi}}^{s}\right) \delta_{\bar{x}}$

where $\bar{\xi}^{s}$ is a set of internal variables, representing the connection failure and $\overline{\overline{\mathbf{K}}}^{\text {s }}$ is set of softening moduli. The yield function for softening is chosen as a multi criteria form, related to, bending, shearing and axial force:

$\dot{\bar{\gamma}}_{i} \overline{\bar{\phi}}_{i}=0 \Rightarrow \overline{\bar{\phi}}^{i}\left(t_{i}, \overline{\bar{q}}_{i}^{s}\right) \leqslant 0$

where $t_{i}$ is traction force and $\overline{\bar{q}}_{i}^{s}$ is stress-like variables, which are work-conjugated to the softening internal variables at the discontinuity for the corresponding failure mode. Among all admissible values of these variables, the principal of maximum dissipation pertinent to softening states will pick the ones that maximize softening dissipation. This can be solved as an unconstrained minimization problem, to provide the evolution equations for internal variables along with the loading/unloading conditions:

$$
\begin{aligned}
& \frac{\partial \overline{\bar{L}}^{s}}{\partial \overline{\overline{\mathbf{q}}}^{s}}=-\dot{\overline{\bar{\xi}}}^{s}+\sum_{1}^{3} \dot{\overline{\bar{\gamma}}} \frac{\partial \overline{\bar{\phi}}}{\partial \overline{\overline{\mathbf{q}}}^{s}}=0 \Rightarrow \dot{\overline{\bar{\xi}}}^{s}=\sum_{1}^{3} \frac{\dot{\bar{\gamma}}_{i} \frac{\partial \overline{\bar{\phi}}}{\partial \overline{\overline{\mathbf{q}}}^{s}}}{\dot{\overline{\boldsymbol{\gamma}}} \geqslant 0, \quad \overline{\bar{\phi}} \leqslant 0, \dot{\bar{\gamma}} \overline{\bar{\phi}}=0}
\end{aligned}
$$

\subsection{Stress resultant form}

By using the rotated strain measure $\mathbf{H}$, we obtain the only nonzero components, defined as:

$H_{11}=\Sigma-\zeta K, H_{21}=\Gamma$

The explicit form of generalized strains can be written as

$$
\begin{aligned}
& \Sigma=H_{11}^{u, v}=\underbrace{\left(1+\frac{d \bar{u}}{d x}\right) \cos \psi+\frac{d \bar{v}}{d x} \sin \psi-1}_{\bar{\Sigma}}+\underbrace{\left(\frac{d \overline{\bar{u}}}{d x} \cos \psi+\frac{d \overline{\bar{v}}}{d x} \sin \psi\right)}_{\overline{\bar{\Sigma}}} \delta_{\bar{x}} \\
& \Gamma=H_{21}^{u, v}=\underbrace{-\left(1+\frac{d \bar{u}}{d x}\right) \sin \psi+\frac{d \bar{v}}{d x} \cos \psi}_{\bar{\Gamma}}+\underbrace{\left(-\frac{d \overline{\bar{u}}}{d x} \sin \psi+\frac{d \overline{\bar{v}}}{d x} \cos \psi\right) \delta_{\bar{x}}}_{\overline{\bar{\Gamma}}} \\
& K=H_{11}^{\psi}=\underbrace{\frac{d \bar{\psi}}{d x}}_{\overline{\bar{K}}}+\underset{\overrightarrow{\bar{K}}}{\frac{d \overline{\bar{\psi}}}{d x}} \delta_{\bar{x}}
\end{aligned}
$$

The linearized strain measures (23) coincide with the strains of the Timoshenko beam [4]. Eq. (23) can be written in matrix compact form: 
$\overline{\boldsymbol{\Sigma}}=\Lambda^{\mathbf{T}}(\mathbf{h}(\overline{\mathbf{a}})-\mathbf{n})+\Lambda^{\mathbf{T}} \mathbf{h}(\overline{\overline{\mathbf{a}}}) \delta_{\bar{x}}$

$\boldsymbol{\Sigma}=\left(\begin{array}{c}\Sigma \\ \Gamma \\ K\end{array}\right), \boldsymbol{\Lambda}=\left[\begin{array}{ccc}\cos \psi & -\sin \psi & 0 \\ \sin \psi & \cos \psi & 0 \\ 0 & 0 & 1\end{array}\right], \mathbf{h}(\overline{\mathbf{a}})=\left(\begin{array}{c}1+\frac{d \bar{u}}{d x} \\ \frac{d \bar{v}}{d x} \\ \frac{d \bar{\psi}}{d x}\end{array}\right)$,

$\mathbf{h}(\overline{\mathbf{a}})=\left(\begin{array}{c}\overline{\bar{u}} \\ \overline{\bar{v}} \\ \overline{\bar{\psi}}\end{array}\right), \mathbf{n}=\Lambda \mathbf{g}_{1}, \mathrm{~g}_{1}=\left(\begin{array}{l}1 \\ 0 \\ 0\end{array}\right)$

By using the same compact notation for the virtual strains (denoted with superposed $(\hat{\bullet})$ ), we can write the weak form of the equilibrium equation, see [4]:

$G(\mathbf{a}, \hat{\mathbf{a}}):=\int_{L}(\hat{\Sigma} N+\hat{\Gamma} V+\hat{K} M) d x-G^{e x t}(\hat{\mathbf{a}})=0$

In (25) above, $N, V$ and $M$ denote stress resultants, expressed in terms of the Biot stress:

$\boldsymbol{\sigma}=(N, V, M)^{T} ; N=\int_{A} T^{11} d A ; V=\int_{A} T^{21} d A ; M=-\int_{A} \zeta T^{11} d A$

The yield function, in the stress resultant form, is defined according to classic works $[11,8]$, except for a small modification to account for isotropic hardening:

$\bar{\phi}(\overline{\boldsymbol{\sigma}}, \overline{\mathbf{q}})=|m|+n^{2}\left(1+v^{2}\right)+v^{4}-1 \leqslant 0$

$m=\frac{M+\bar{q}_{M}}{M_{y}} ; v=\frac{V_{V}+\bar{q}_{V}}{V_{y}} ; n=\frac{N+\bar{q}_{N}}{N_{y}}$

where $m$ is a non-dimensional bending moment; $v$ is a nondimensional shear force; $n$ is a non-dimensional axial force; $\bar{q}_{M}, \bar{q}_{V}, \bar{q}_{N}$ are internal hardening stress like variables; whereas $M_{y}$, $V_{y}$ and $N_{y}$ denote yield bending moment, yield shear force and yield axial force. The yield function for softening is chosen as a multi criteria form, pertaining to, bending moment, shear and axial force:

$$
\dot{\overline{\bar{\gamma}}} \overline{\bar{\phi}}_{i}=0 \Rightarrow \begin{aligned}
& \overline{\bar{\phi}}^{M}\left(t_{M}, \overline{\bar{q}}_{M}^{s}\right)=\left|t_{M}\right|-\left(t_{M_{y}}-\overline{\bar{q}}_{M}^{s}\right) \leqslant 0 \\
& \overline{\bar{\phi}}^{V}\left(t_{V}, \overline{\bar{q}}_{V}^{s}\right)=\left|t_{V}\right|-\left(t_{V_{y}}-\overline{\bar{q}}_{V}^{s}\right) \leqslant 0 \\
& \overline{\bar{\phi}}^{N}\left(t_{N}, \overline{\bar{q}}_{N}^{s}\right)=\left|t_{N}\right|-\left(t_{N_{y}}-\overline{\bar{q}}_{N}^{s}\right) \leqslant 0
\end{aligned}
$$

where $t_{M}, t_{V}, t_{N}$ are traction forces, $t_{M_{y}}, t_{V_{y}}, t_{N_{y}}$ are the corresponding ultimate values where softening starts and $\overline{\bar{q}}_{M}^{s}, \overline{\bar{q}}_{V}^{s}, \overline{\bar{q}}_{N}^{s}$ are stress-like variables work-conjugate to softening variables at the discontinuity.

\subsection{Consistent linearization of virtual work equations}

As shown in (7) [4], the virtual work equation can be expressed in terms of different stress- strain energy-conjugated pairs. Any of them leads to a nonlinear problem, which requires an iterative solution procedure. With Newton's iterative method, we need to perform consistent linearization at each iteration.

\subsubsection{Incompatible modes implementation}

The embedded discontinuity formulation that handles the softening is implemented in the framework of incompatible modes [5]. Namely, we turn to Hu-Washizu variational formulation, where the weak form is constructed for all three groups of equations: kinematics, constitutive and equilibrium equations. Namely, we choose the spaces of virtual displacements, virtual stress and virtual strain to write:

$$
\begin{aligned}
& \mathbf{G}_{\mathbf{a}}(\mathbf{u}, \mathbf{H}, \mathbf{T} ; \hat{\mathbf{u}}):=\int_{L} \int_{A} \hat{\mathbf{H}} \mathbf{T} d A d x-\int_{L} \hat{\mathbf{u}}^{\mathrm{T}} \mathbf{f} d x=0 \\
& \mathbf{G}_{\mathbf{r}}(\mathbf{u}, \mathbf{H}, \mathbf{T} ; \hat{\mathbf{T}}):=\int_{L} \int_{A} \hat{\mathbf{T}} \overline{\bar{H}} \mathbf{d} \mathbf{A} d x=0 \\
& \mathbf{G}_{\hat{\mathbf{z}}}(\mathbf{u}, \mathbf{H}, \mathbf{T} ; \hat{\mathbf{H}}):=\int_{L} \int_{A} \hat{\mathbf{H}}\left(\mathbf{C}^{e p} \mathbf{H}-\mathbf{T}\right) d A d x=0
\end{aligned}
$$

where virtual fields are denoted with superposed $(\hat{\bullet}) ; \hat{\mathbf{H}}$ - virtual rotated strain field; $\hat{\mathbf{T}}$ - virtual stress field and $\hat{\mathbf{u}}$ - virtual displacements field. Virtual rotated strain measure can be derived by taking the directional derivative of strain measure $\mathbf{U}(11)$ and exploiting relation $\mathbf{U}=\mathbf{H}+\mathbf{I}$ :

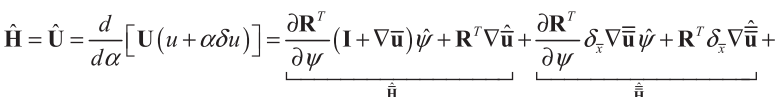

$$
\begin{aligned}
& +\underbrace{\frac{\partial \mathbf{R}^{T}}{\partial \psi}(\mathbf{I}+\nabla \bar{\psi}) \hat{\psi}+\mathbf{R}^{T} \nabla \hat{\bar{\psi}}}_{\hat{\hat{\mathbf{H}}}_{\psi}}+\underbrace{\frac{\partial \mathbf{R}^{T}}{\partial \psi} \delta_{\bar{x}} \nabla \overline{\bar{\psi}} \hat{\psi}+\mathbf{R}^{T} \delta_{\bar{x}} \nabla \hat{\bar{\psi}}}_{\hat{\overline{\mathbf{H}}}_{\psi}}
\end{aligned}
$$

In Eq. (30), the additive decomposition of the displacement gradient field produces an additive decomposition of the virtual strain measure $\hat{\mathbf{H}}$. The virtual stress field can be expressed as:

$\hat{\mathbf{T}}=\mathbf{C}^{e p}\left(\hat{\overline{\mathbf{H}}}+\delta_{\overline{\bar{x}}} \hat{\overline{\mathbf{H}}}\right)$

By exploiting results (22)-(25) and (29)-(31) we can construct the weak form of equilibrium equations in terms of stress resultants:

$$
\begin{aligned}
\mathbf{G}_{\mathbf{a}}(\mathbf{a}, \boldsymbol{\Sigma}, \mathbf{a} ; \hat{\mathbf{a}}):= & \int_{L}\left(\mathbf{d}^{\mathbf{T}}(\overline{\mathbf{a}}) \Lambda+\bar{\psi} \mathbf{h}^{\mathbf{T}}(\mathbf{a}) \frac{d \mathbf{\Lambda}}{d \psi}\right) \mathbf{C}^{\mathbf{e p}} \Lambda^{\mathbf{T}}(\mathbf{h}(\mathbf{a})-\mathbf{n}) d x \\
& -\int_{L} \hat{\mathbf{a}}^{\mathbf{T}} \mathbf{f} d x=0 \\
\mathbf{G}_{\mathbf{r}}(\mathbf{a}, \boldsymbol{\Sigma}, \mathbf{r} ; \hat{\mathbf{r}}):= & \int_{L} \hat{\mathbf{r}}(\overline{\boldsymbol{\Sigma}}-\boldsymbol{\Sigma}) d x=0 \\
\mathbf{G}_{\hat{\mathbf{\Sigma}}}(\mathbf{a}, \boldsymbol{\Sigma}, \mathbf{r} ; \hat{\mathbf{\Sigma}}):= & \int_{L} \hat{\mathbf{\Sigma}}\left(\mathbf{C}^{e p} \boldsymbol{\Sigma}-\mathbf{r}\right) d x=0
\end{aligned}
$$

The virtual strain measure $\hat{\boldsymbol{\Sigma}}$ can be derived by taking the directional derivative of the strain measures in (23), which can be written explicitly as:

$\hat{\bar{\Sigma}}=\frac{d \hat{\bar{u}}}{d x} \cos \psi+\frac{d \hat{\bar{v}}}{d x} \sin \psi+\hat{\psi}\left[-\left(1+\frac{d \bar{u}}{d x}\right) \sin \psi+\frac{d \bar{v}}{d x} \cos \psi\right]$

$\hat{\overline{\bar{\Sigma}}} \delta_{\bar{x}}=\hat{\overline{\bar{u}}} \delta_{\bar{x}} \cos \psi+\hat{\overline{\bar{v}}} \delta_{\bar{x}} \sin \psi+\hat{\psi}[-\hat{\overline{\bar{u}}} \sin \psi+\hat{\overline{\bar{v}}} \cos \psi] \delta_{\bar{x}}$

$\hat{\bar{\Gamma}}=-\frac{d \hat{\bar{u}}}{d x} \sin \psi+\frac{d \hat{\bar{v}}}{d x} \cos \psi+\hat{\psi}\left[\left(1+\frac{d \bar{u}}{d x}\right) \cos \psi-\frac{d \bar{v}}{d x} \sin \psi\right]$

$\hat{\overline{\bar{\Gamma}}} \delta_{\bar{x}}=-\hat{\overline{\bar{u}}} \delta_{\bar{x}} \sin \psi+\hat{\bar{v}} \delta_{\bar{x}} \cos \psi+\hat{\psi}[\hat{\bar{u}} \cos \psi-\hat{\overline{\bar{v}}} \sin \psi] \delta_{\bar{x}}$

$\hat{\bar{K}}=\frac{d \hat{\bar{\psi}}}{d x} ; \hat{\bar{K}} \delta_{\bar{x}}=\hat{\bar{\psi}} \delta_{\bar{x}}$

The virtual strains components (33) can be put in the matrix form as

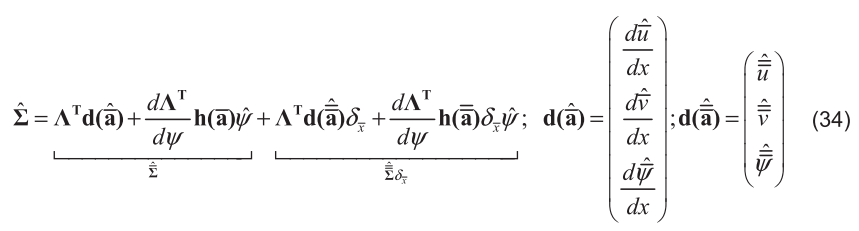

The discrete form can be obtained at the later stage, given that the linearization and discretization commute. The weak form of the virtual work equation can be expressed as:

$\mathbf{G}(\mathbf{a}, \hat{\mathbf{a}}):=\int_{L} \hat{\boldsymbol{\Sigma}} \cdot \boldsymbol{\sigma} d x-\int_{L} \hat{\mathbf{a}}^{\mathrm{T}} \mathbf{f} d x=0$ 
where $\mathbf{a}$ is a vector real displacements, $\hat{\mathbf{a}}$ is a vector of virtual displacements; $\hat{\boldsymbol{\Sigma}}$ is a virtual strain measure; $\boldsymbol{\sigma}$ is a vector of stress resultant forces and $\mathbf{f}$ is a vector of external forces.The stress resultant forces $\boldsymbol{\sigma}$ for the elastoplastic response can be written as

$\boldsymbol{\sigma}=\mathbf{C}^{e p} \boldsymbol{\Lambda}^{\mathbf{T}}(\mathbf{h}(\mathbf{a})-\mathbf{n}) ; \mathbf{C}=\operatorname{diag}\left(C_{N}^{e p}, C_{V}^{e p}, C_{M}^{e p}\right)$

where $C_{N}^{e p}, C_{V}^{e p}, C_{M}^{e p}$ are elastoplastic stiffnesses of the beam section for an axial force, shear force and bending moment.

By enforcing the orthogonality condition [3] for the element with incompatible modes, which results in elimination of the stress field and allows us to write the remaining set of equilibrium equations (32) as

$\mathbf{G}_{\mathbf{a}}(\mathbf{a}, \boldsymbol{\Sigma}, \mathbf{a} ; \hat{\mathbf{a}}):=\int_{L} \hat{\bar{\Sigma}} \mathbf{C}^{\mathbf{e p}}\left(\bar{\Sigma}+\overline{\bar{\Sigma}} \delta_{\bar{x}}\right) d x-\int_{L} \hat{\mathbf{a}}^{\mathbf{T}} \mathbf{f} d x=0$

$\mathbf{G}_{\hat{\mathbf{\Sigma}}}(\mathbf{a}, \boldsymbol{\Sigma}, \mathbf{r} ; \hat{\mathbf{\Sigma}}):=\int_{L} \hat{\overline{\bar{\Sigma}}} \mathbf{C}^{e p}\left(\overline{\boldsymbol{\Sigma}}+\overline{\overline{\mathbf{\Sigma}}} \delta_{\bar{x}}\right) d x=0$

In the last expression, the virtual strains are obtained explicitly by directional derivative computation:

$$
\begin{aligned}
& \hat{\boldsymbol{\Sigma}}=\left.(d / d \beta)[\tilde{\overline{\mathbf{\Sigma}}}(\tilde{\overline{\mathbf{a}}}+\boldsymbol{\beta} \hat{\overline{\mathbf{a}}})+\overline{\overline{\mathbf{\Sigma}}}(\overline{\overline{\mathbf{a}}}+\boldsymbol{\beta} \hat{\overline{\overline{\mathbf{a}}}})]\right|_{\beta=0} \\
& =\underbrace{\boldsymbol{\Lambda}^{\mathrm{T}} \mathbf{d}(\hat{\mathbf{a}})+\frac{d \boldsymbol{\Lambda}^{\mathrm{T}}}{d \psi} \mathbf{h}(\overline{\mathbf{a}}) \hat{\bar{\psi}}+\boldsymbol{\Lambda}^{\mathrm{T}} \mathbf{d}(\hat{\overline{\mathbf{a}}}) \delta_{\bar{x}}+\frac{d \boldsymbol{\Lambda}^{\mathrm{T}}}{d \psi} \mathbf{h}(\overline{\overline{\mathbf{a}}}) \delta_{\overline{\bar{x}}} \hat{\bar{\psi}}}_{\text {玄 }}
\end{aligned}
$$

In order to provide the quadratic convergence of Newton's method, we need to find the consistent tangent stiffness. The latter can be obtained by consistent linearization of the weak form in $(37)_{1}$ resulting with

$$
\begin{aligned}
\left.L[G]\right|_{\mathbf{a}}= & \left.\mathbf{G}_{\mathbf{a}}(\mathbf{a}, \boldsymbol{\Sigma}, \mathbf{a} ; \hat{\overline{\mathbf{a}}})\right|_{a}+\left.\frac{d}{d \beta}[G(\hat{\overline{\mathbf{a}}}, \mathbf{a}+\beta \Delta \mathbf{a})]\right|_{\beta=0} \\
= & G(\mathbf{a}, \hat{\mathbf{a}})+\int_{L}(\mathbf{d}(\hat{\overline{\mathbf{a}}}) ; \hat{\bar{\psi}})\left[\mathbf{D}_{m}^{K}+\mathbf{D}_{g}^{K}\right]\left(\begin{array}{c}
\mathbf{d}(\Delta \overline{\mathbf{a}}) \\
\Delta \bar{\psi}
\end{array}\right) d x \\
& +\int_{L}(\mathbf{d}(\hat{\overline{\mathbf{a}}}) ; \hat{\bar{\psi}})\left[\mathbf{D}_{m}^{F}\right]\left(\begin{array}{c}
\mathbf{d}(\Delta \overline{\overline{\mathbf{a}}}) \\
\Delta \overline{\bar{\psi}}
\end{array}\right) d x=0
\end{aligned}
$$

where $\mathbf{D}_{m}^{k}$ and $\mathbf{D}_{g}^{k}$ are defined in (42), along with

$$
\begin{aligned}
& \left.L[G]\right|_{\Sigma}=\left.G(\mathbf{a}, \hat{\mathbf{\Sigma}})\right|_{\Sigma}+\left.\frac{d}{d \beta}[G(\hat{\mathbf{\Sigma}}, \mathbf{a}+\beta \Delta \mathbf{a})]\right|_{\beta=0} \\
& =G(\mathbf{a}, \hat{\boldsymbol{\Sigma}})+\int_{L}(\mathbf{d}(\hat{\overline{\mathbf{a}}}) ; \hat{\bar{\psi}})\left[\mathbf{D}_{m}^{F}\right]\left(\begin{array}{c}
\mathbf{d}(\Delta \mathbf{a}) \\
\Delta \psi
\end{array}\right) d x \\
& +\int_{L}(\mathbf{d}(\hat{\overline{\mathbf{a}}}) ; \hat{\bar{\psi}})\left[\mathbf{D}_{m}^{H}+\mathbf{D}_{g}^{H}\right]\left(\begin{array}{c}
\mathbf{d}(\Delta \overline{\overline{\mathbf{a}}}) \\
\Delta \overline{\bar{\psi}}
\end{array}\right) d x=0
\end{aligned}
$$

where

$\mathbf{D}_{m}^{F}=\mathbf{D}_{m}^{H}=\left[\begin{array}{c}\boldsymbol{\Lambda} \\ \mathbf{h}^{\mathbf{T}}(\overline{\overline{\mathbf{a}}}) \frac{d \Lambda}{d \psi}\end{array}\right] \mathbf{C}^{\mathbf{e p}}\left[\boldsymbol{\Lambda}^{\mathbf{T}} ; \frac{d \Lambda^{\mathbf{T}}}{d \psi} \mathbf{h}(\overline{\overline{\mathbf{a}}})\right] ; \mathbf{D}_{g}^{H}=\left[\begin{array}{cc}0 & \frac{d \Lambda}{d \psi} \mathbf{r} \\ \mathbf{r}^{\mathbf{T}} \frac{d \Lambda^{\mathbf{T}}}{d \psi} & \mathbf{h}^{\mathbf{T}}(\overline{\overline{\mathbf{a}}}) \frac{d^{2} \Lambda}{d \psi^{2}} \mathbf{r}\end{array}\right]$

$\mathbf{D}_{m}^{k}=\left[\begin{array}{c}\boldsymbol{\Lambda} \\ \mathbf{h}^{\mathbf{T}}(\mathbf{a}) \frac{d \boldsymbol{\Lambda}}{d \psi}\end{array}\right] \mathbf{C}^{\mathbf{e p}}\left[\boldsymbol{\Lambda}^{\mathbf{T}} ; \quad \frac{d \Lambda^{\mathbf{T}}}{d \psi} \mathbf{h}(\mathbf{a})\right] ; \mathbf{D}_{g}^{k}=\left[\begin{array}{cc}0 & \frac{d \boldsymbol{\Lambda}}{d \psi} \mathbf{r} \\ \mathbf{r}^{\mathbf{T}} \frac{d \Lambda^{\mathbf{T}}}{d \psi} & \mathbf{h}^{\mathbf{T}}(\mathbf{a}) \frac{d^{2} \Lambda}{d \psi^{2}} \mathbf{r}\end{array}\right]$

\section{Finite element approximation}

We choose the simplest finite element approximation for the presented beam model with plasticity that fits within the frame- work of incompatible modes method. We here provide some details of numerical implementation for a beam element with two nodes and three localized failure modes. We allow for displacement discontinuity representation for bending moment, shear force and axial force, each with an additional parameter $\boldsymbol{\alpha}_{e}$ :

$$
\begin{aligned}
& x(\xi)=\sum_{a=1}^{2} N_{a}(\xi) \cdot x_{a} ; N_{a}(\xi)=\frac{1}{2}\left(1+\xi_{a} \xi\right) \\
& u^{h}\left(\xi, t_{n+1}\right)=\sum_{a=1}^{2} N_{a}(\xi) \cdot u_{a}(t)+M^{e}(\xi) \alpha_{u}^{e}(t) ; \\
& v^{h}\left(\xi, t_{n+1}\right)=\sum_{a=1}^{2} N_{a}(\xi) \cdot v_{a}(t)+M^{e}(\xi) \alpha_{v}^{e}(t) ; \\
& \psi^{h}\left(\xi, t_{n+1}\right)=\sum_{a=1}^{2} N_{a}(\xi) \cdot \psi_{a}(t)+M^{e}(\xi) \alpha_{\psi}^{e}(t) ; \\
& M^{e}(\xi)= \begin{cases}-N_{2}(\xi)=-\frac{1}{2}(1+\xi), & \text { for } \xi \in[-1,0] \\
H_{\xi}(\xi)-N_{2}(\xi)=\frac{1}{2}(1-\xi), & \text { for } \xi \in[0,1]\end{cases}
\end{aligned}
$$

where $\xi \in[-1,1]$ is natural coordinate at the parent element and $H_{\bar{\xi}}(\xi)$ is Heaviside function related to the point $\bar{\xi}=\xi=0$. The two-node element interpolation is enhanced with the displacement discontinuity, placed in the center of this element. The corresponding approximation of displacements gradient can then be written as:

$$
\begin{aligned}
& \frac{d u^{h}(\xi, t)}{d x}=\sum_{a=1}^{2} B_{a}(\xi) u_{a}(t)+G^{e}(\xi) \alpha_{u}^{e}(t) \\
& \frac{d v^{h}(\xi, t)}{d x}=\sum_{a=1}^{2} B_{a}(\xi) v_{a}(t)+G^{e}(\xi) \alpha_{v}^{e}(t) \\
& \frac{d \psi^{h}(\xi, t)}{d x}=\sum_{a=1}^{2} B_{a}(\xi) \psi_{a}(t)+G^{e}(\xi) \alpha_{\psi}^{e}(t)
\end{aligned}
$$

where

$$
\begin{aligned}
& B_{a}(\xi)=\frac{d N_{a}(\xi)}{d x} ; B_{a}(\xi)=\frac{(-1)^{a}}{l^{e}} \\
& G^{e}(\xi)=\frac{d M_{a}(\xi)}{d x} ; G^{e}(\xi)= \begin{cases}-\frac{1}{l^{e}}, & \text { for } \xi \in[-1,0) \cup(0,1] \\
-\frac{1}{l^{e}}+\delta_{0}, & \text { for } \xi=0\end{cases}
\end{aligned}
$$

with $\delta_{0}$ the Dirac delta function placed in the center of the element. This choice will ensure that the incompatible mode variation remains orthogonal to the constant stress in each element.

By combining the results in (24), (43) and (44), we can construct strain field approximation. We typically use reduced numerical integration with a single point, $\xi=0$, in order to avoid locking phenomena [4]:

$$
\begin{aligned}
\Sigma^{h}(0, t) & =\left(\sum_{a=1}^{2} B_{a}(0) \cdot u_{a}(t)+G^{e}(0) \alpha_{u}^{e}(t)\right) \cos (\sum_{a=1}^{2} N_{a}(0) \cdot \psi_{a}(t)+\underbrace{M^{e}(0) \alpha_{\psi}^{e}}_{=0}(t)) \\
& +\left(\sum_{a=1}^{2} B_{a}(0) \cdot v_{a}(t)+G^{e}(0) \alpha_{v}^{e}(t)\right) \sin (\sum_{a=1}^{2} N_{a}(0) \cdot \psi_{a}(t)+\underbrace{M^{e}(0) \alpha_{\psi}^{e}(t)}_{=0}) \\
\Gamma^{h}(0, t) & =\left(\sum_{a=1}^{2} B_{a}(0) \cdot u_{a}(t)+G^{e}(0) \alpha_{u}^{e}(t)\right) \sin (\sum_{a=1}^{2} N_{a}(0) \cdot \psi_{a}(t)+\underbrace{M^{e}(0) \alpha_{\psi}^{e}(t)}_{=0}) \\
& +\left(\sum_{a=1}^{2} B_{a}(0) \cdot v_{a}(t)+G^{e}(0) \alpha_{v}^{e}(t)\right) \cos (\sum_{a=1}^{2} N_{a}(0) \cdot \psi_{a}(t)+\underbrace{M^{e}(0) \alpha_{\psi}^{e}(t)}_{=0}) \\
& -\sum_{a=1}^{2} N_{a}(0) \cdot \psi_{a}(t) \\
K^{h}(0, t) & =\sum_{a=1}^{2} B_{a}(0) \cdot \psi_{a}(t)+G^{e}(0) \alpha_{\psi}^{e}(t)
\end{aligned}
$$


We note that a pure bending deformation mode (Kirchoff's constraint), which imposes that both shear and membrane deformations are equal to zero $\left(\Sigma^{h}(\xi)=\Gamma^{h}(\xi)=0, \forall \xi\right)$, can be obtained only if we have one point of integration and $M^{e}(x) \dot{\alpha}_{\psi}^{e}(t)=0$. The stress field approximation can be obtained from the regular part of the strain rate in (45) with no contribution from the singular part, which represents softening plastic strain rate. We can write:

$$
\begin{aligned}
& N^{h}\left(0, t_{n+1}\right)=E A\left(\tilde{\bar{\Sigma}}^{h}(0, t)+G^{e}(0) \overline{\bar{\Sigma}}^{h}(t)\right) \\
& V^{h}\left(0, t_{n+1}\right)=G A\left(\widetilde{\Gamma}^{h}(0, t)+G^{e}(0) \overline{\bar{\Gamma}}^{h}(t)\right) \\
& M^{h}\left(0, t_{n+1}\right)=E I\left(\sum_{a=1}^{2} B_{a}(0) \cdot \psi_{a}(t)+G^{e}(0) \alpha_{\psi}^{e}(t)\right)
\end{aligned}
$$

where

$$
\begin{aligned}
& \overline{\bar{\Sigma}}^{h}(t)=\alpha_{u}^{e}(t) \cos \left(\sum_{a=1}^{2} N_{a}(0) \cdot \psi_{a}(t)\right)+\alpha_{v}^{e}(t) \sin \left(\sum_{a=1}^{2} N_{a}(0) \cdot \psi_{a}(t)\right) \\
& \overline{\bar{\Gamma}}^{h}(t)=-\alpha_{u}^{e}(t) \sin \left(\sum_{a=1}^{2} N_{a}(0) \cdot \psi_{a}(t)\right)+\alpha_{v}^{e}(t) \cos \left(\sum_{a=1}^{2} N_{a}(\xi) \cdot \psi_{a}(t)\right)
\end{aligned}
$$

\section{Numerical examples}

Several numerical examples are presented in this section to illustrate the performance of the proposed finite element formula- tion. All numerical computations are performed with a research version of the computer program FEAP [12].

\subsection{Straight cantilever under imposed end rotation}

In this example, we present three different types of a response for a cantilever beam under free-end bending load. The geometric properties of the cross section correspond to standard hot rolled profile IPE 200 and material properties take values for steel class S235. The initially straight cantilever beam model is constructed with three different meshes of 2, 4 and 8 elements. Each analysis is performed under imposed end rotation $\psi=\pi$. The first analysis represents the linear elastic response (see Fig. 1), the second analysis represents the elastoplastic response that remains in hardening phase (see Fig. 2), whereas the third analysis represents the elastoplastic response that goes into the softening phase, failure. The failure is localized in the middle of the cantilever, where one element is weakened (see Fig. 3). Response diagrams show the mesh indifference of the proposed formulation.

For the chosen properties of the cantilever (Young's modulus: $E=2 \cdot 10^{4} \mathrm{kN} / \mathrm{cm}^{2}$; Hardening modulus: $\mathrm{K}=0.05 \cdot \mathrm{E}$; Moment of inertia: $I=1940 \mathrm{~cm}^{4}$; Area of the cross section: $A=28.5 \mathrm{~cm}^{2}$; Yield bending moment: $M_{y}=3100 \mathrm{kN} \mathrm{cm}$ ), some of the results can be verified analytically. Namely, the elastic bending moment can be computed as $\mathrm{M}_{\mathrm{e}}=\pi \cdot E I / L=1218320 \mathrm{kN} \mathrm{cm}$ and the elastoplastic bending moment as $\mathrm{M}_{\mathrm{ep}}=\left(\pi-K_{y}\right) \cdot E K /(E+K) L+K_{y} \cdot E I / L=$ $9145,87 \mathrm{kN} \mathrm{cm}$. The comparison, these reference values versus numerical results computed with different number of elements, is presented in Table 1.
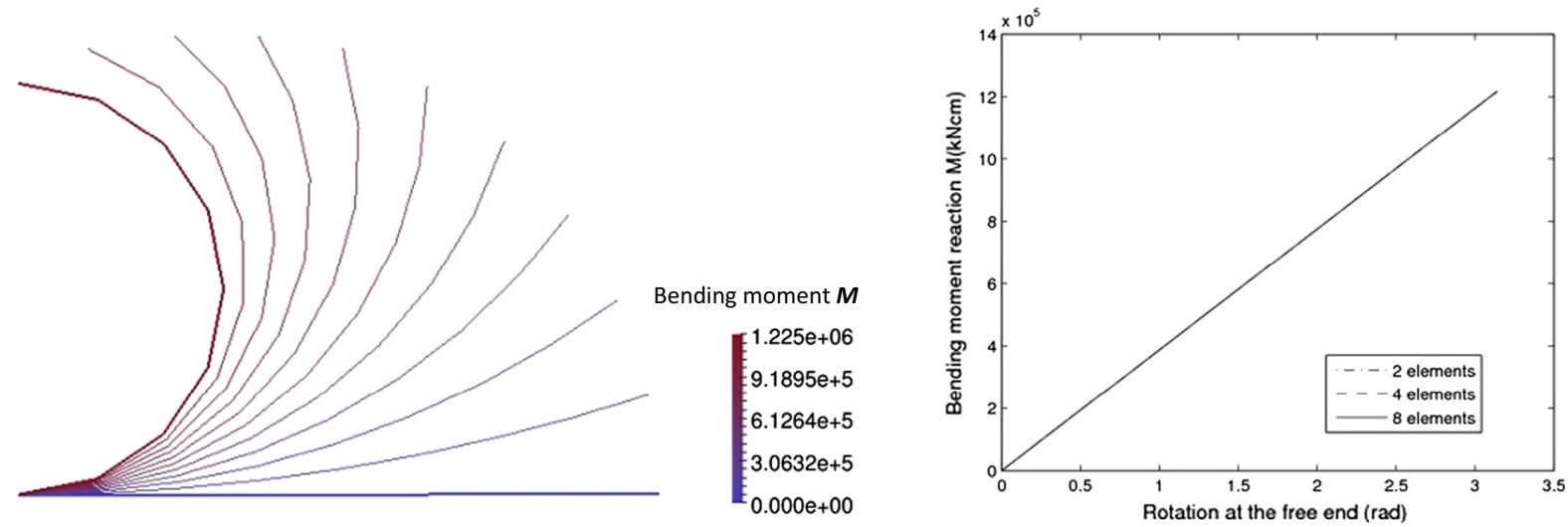

Fig. 1. Linear elastic analysis: deformed configuration and diagram $(M[\mathrm{kN} \mathrm{cm}]-\psi[\mathrm{rad}])$.
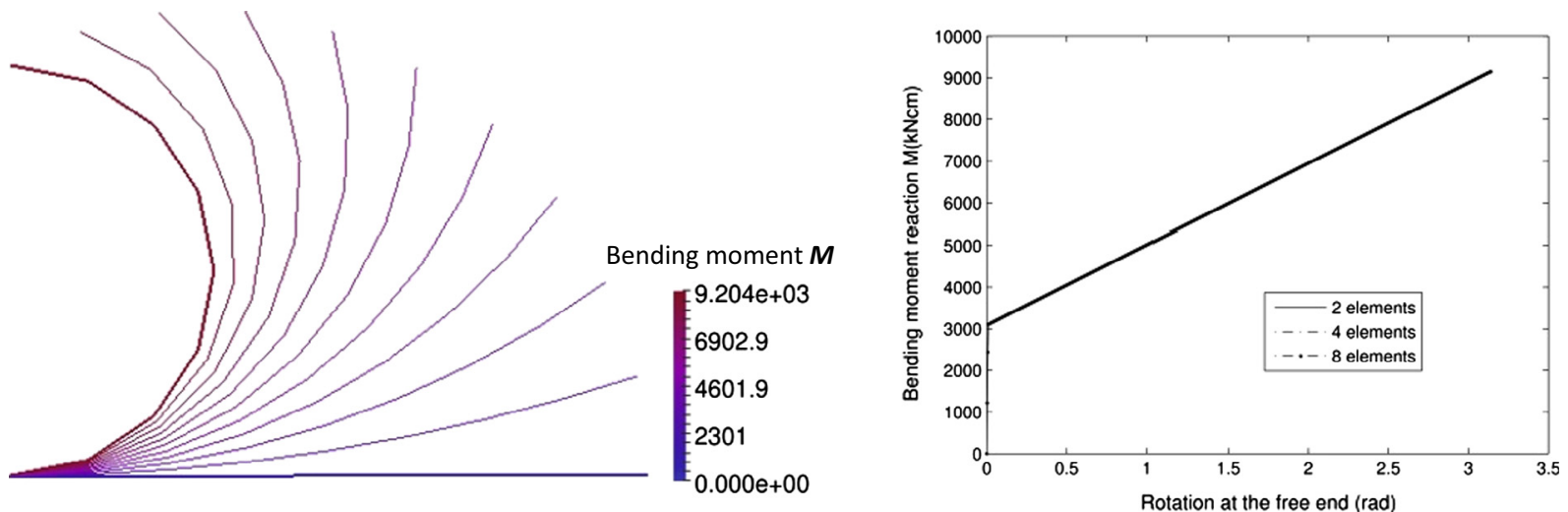

Fig. 2. Elastic-plastic analysis: deformed configuration and diagram $(M[\mathrm{kN} \mathrm{cm}]-\psi[\mathrm{rad}])$ 

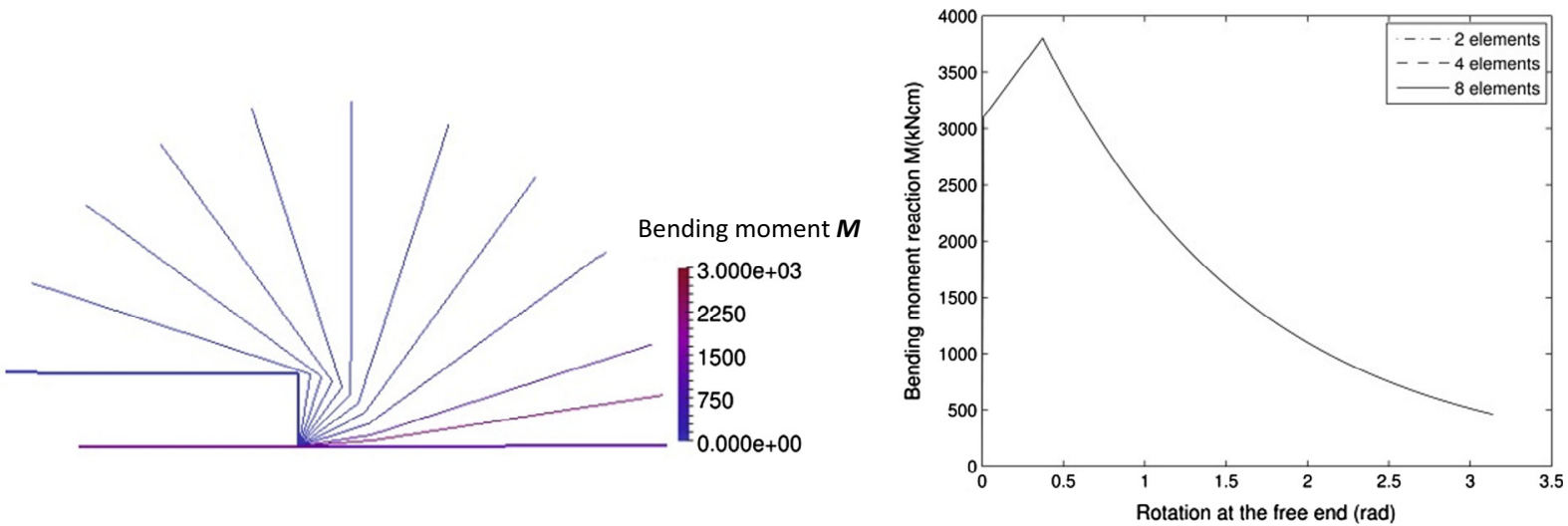

Fig. 3. Ultimate limit analysis with included failure: deformed configuration and response curve.

Table 1

Cantilever beam under imposed an end rotation.

\begin{tabular}{lll}
\hline \multirow{2}{*}{ No. of elements } & \multicolumn{2}{l}{ Bending moment } \\
\cline { 2 - 3 } & Elastic analysis $(\mathrm{kN} \mathrm{cm})$ & Elastoplastic anal. $(\mathrm{kN} \mathrm{cm})$ \\
\hline 2 & $1,218,300$ & 9146 \\
4 & $1,218,300$ & 9146 \\
8 & $1,218,300$ & 9146 \\
16 & $1,218,300$ & 9146 \\
Exact & $1,218,320$ & 9145,87 \\
\hline
\end{tabular}

\subsection{Straight cantilever under imposed free-end vertical displacement}

This example presents two different failure modes under freeend vertical displacement. Namely, by imposing vertical displacement at the free end of a cantilever, we can trigger failure due to either bending moment or shearing force. The type of failure depends on chosen values for constitutive parameters. We first perform analysis (see Fig. 4) where the ultimate bending $M_{u}$ is reached before the ultimate shear force $V_{u}\left(M_{u}=3800 \mathrm{kN} \mathrm{cm}\right.$, $\left.\mathrm{V}_{\mathrm{u}}=75 \mathrm{kN}\right)$. We then modify the parameters $\left(\mathrm{M}_{\mathrm{u}}=3800 \mathrm{kN} \mathrm{cm}\right.$, $\mathrm{V}_{\mathrm{u}}=65 \mathrm{kN}$ ), see Fig. 5 , in the second analysis, in order to reach the ultimate shear force before the ultimate bending moment. In Table 2, we provide the results of studies for the typical rate of convergence.

\subsection{Push-over analysis of a symmetric frame}

In this example, we present the results for a push-over analysis of symmetric steel frame. The frame geometry is given in Fig. 6. The material properties for all frame members are equal (Young's modulus: $E=2 \cdot 10^{4} \mathrm{kN} / \mathrm{cm}^{2}$; Hardening modulus: $\mathrm{K}=0.05 \mathrm{E}$; Moment of inertia: $I=1940 \mathrm{~cm}^{4}$; Area of the cross section: $A=28.5 \mathrm{~cm}^{2}$; Yield bending moment: $M_{y}=3100 \mathrm{kN} \mathrm{cm}$; Ultimate
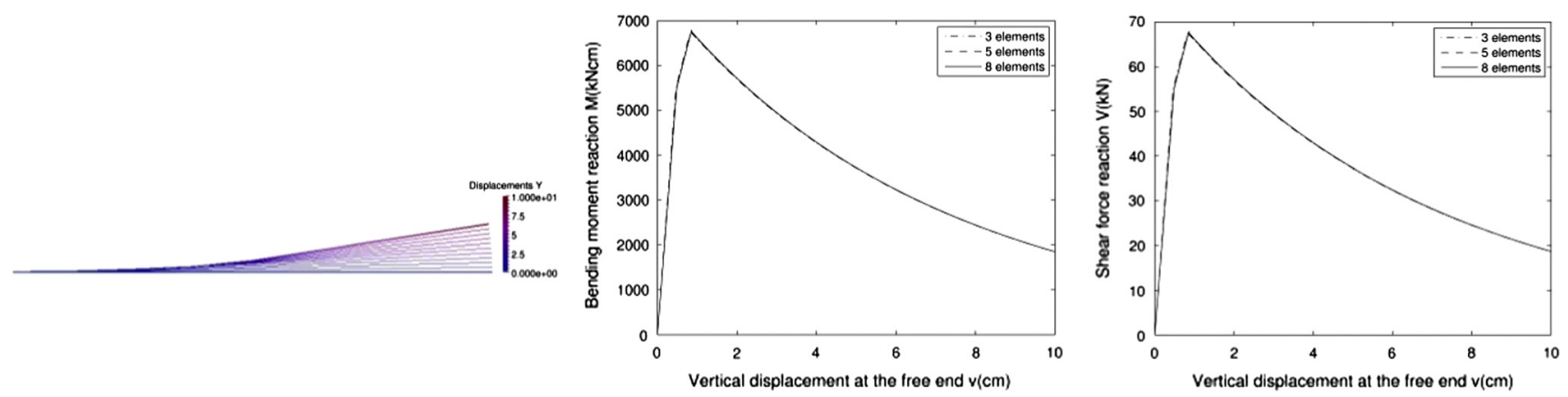

Fig. 4. Failure in bending: deformed configuration and response curves.
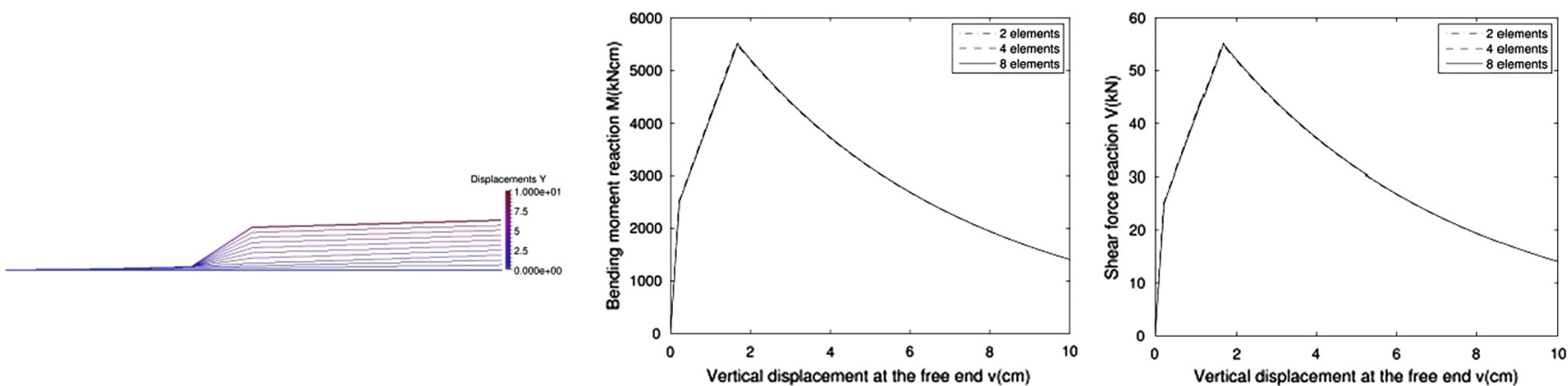

Fig. 5. Failure in shearing: deformed configuration and response curves. 
Table 2

Reduction in residual and energy norm in one increment (softening).

\begin{tabular}{|c|c|c|c|c|}
\hline \multirow[t]{2}{*}{ No. of iterations } & \multicolumn{2}{|c|}{ Failure in the bending } & \multicolumn{2}{|c|}{ Failure in the shearing } \\
\hline & Residual & Energy & Residual & Energy \\
\hline 1 & $2.5451184 \mathrm{E}+03$ & $2.89262392986 \mathrm{E}+00$ & $2.5617356 \mathrm{E}+03$ & $2.88022968875 \mathrm{E}+00$ \\
\hline 2 & $1.2603427 \mathrm{E}-02$ & 7.72176817049E-09 & $2.2358020 \mathrm{E}-01$ & $2.94749460179 \mathrm{E}-08$ \\
\hline 3 & $3.1269310 \mathrm{E}-10$ & $5.37722363293 \mathrm{E}-25$ & $5.5411964 \mathrm{E}-05$ & $2.27071800263 \mathrm{E}-14$ \\
\hline 4 & & & $1.0282664 \mathrm{E}-07$ & $2.31118241048 \mathrm{E}-20$ \\
\hline
\end{tabular}

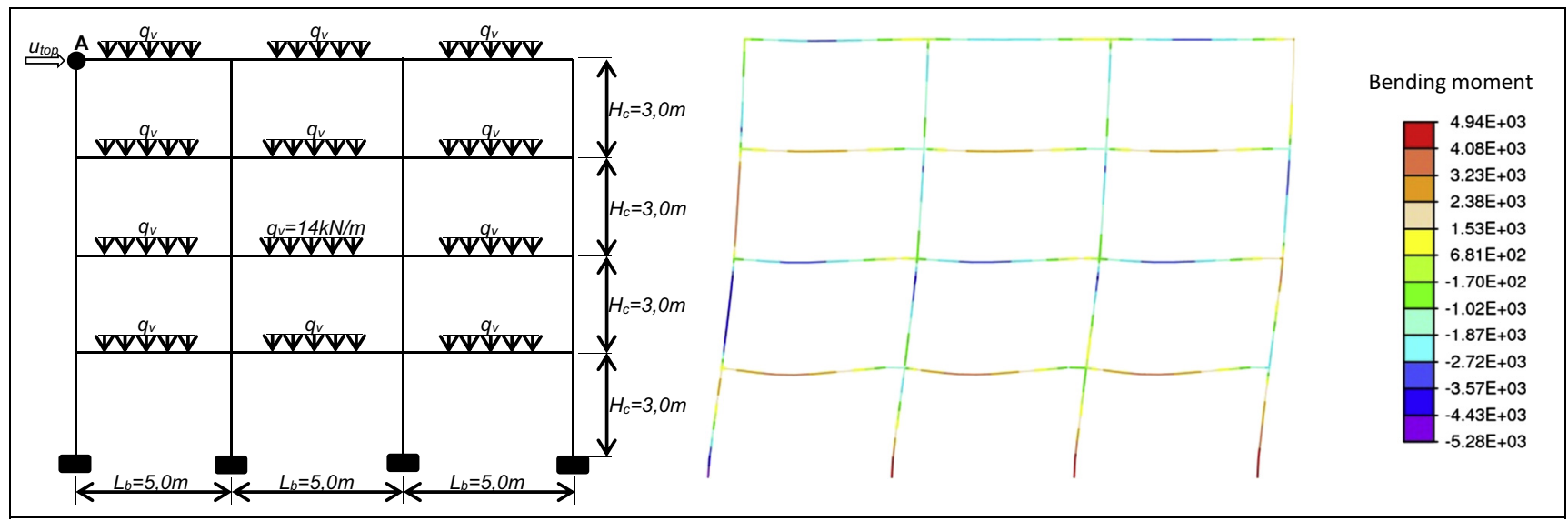

Fig. 6. (a) Frame geometry and loading and (b) deformed shape and bending moment distribution.
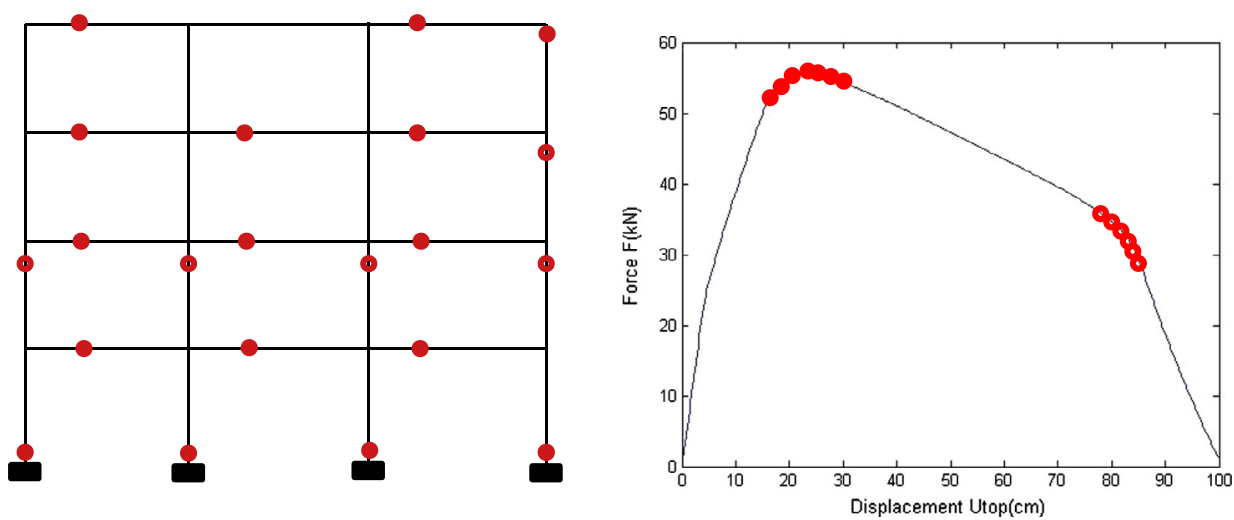

Fig. 7. Locations of softening plastic hinges and load versus displacement $\left(u_{\text {top }}=100 \mathrm{~cm}\right)$.

bending moment: $M_{u}=3100 \mathrm{kN} \mathrm{cm}$; Yield shear force: $\mathrm{V}_{\mathrm{y}}=355 \mathrm{kN}$; Ultimate shear force: $V_{u}=400 \mathrm{kN}$, Fracture energies: $G_{f, M}=550$ and $\left.G_{f, V}=450\right)$, except the fact that the cross-section properties of the columns are $10 \%$ stronger then cross-section properties of the beams. The elements which connect beams to columns are $10 \%$ weaker than cross-section properties of beams; these elements are chosen to simulate the behavior of connections in the global analysis of the steel frame structure. The vertical load was applied to all beam members. This load is kept constant throughout pushover analysis in order to simulate the dead load effect. The lateral loading is applied in terms of an imposed incremental displacement $\left(u_{\text {top }}\right)$ at the upper corner (point A, see Fig. 6). In Fig. 6b, we present the deformed configuration of the steel frame and the corresponding distribution of the bending moments. In Fig. 7, we present the position of activated plastic hinges in the final stage of failure, along with the computed softening response in terms of the force-displacement diagram. In Fig. 7b, the force denotes reaction in the corner $\mathrm{A}$, where is imposed the displacement.

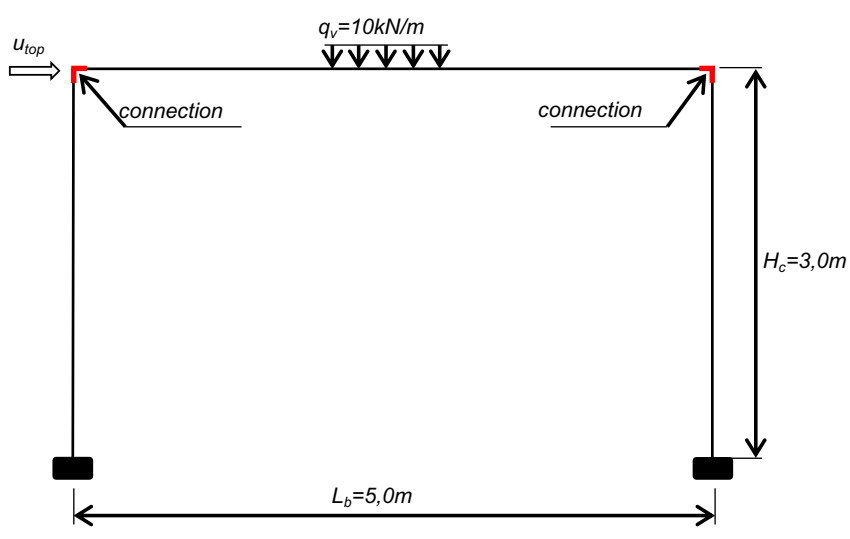

Fig. 8. Frame geometry and loading. 


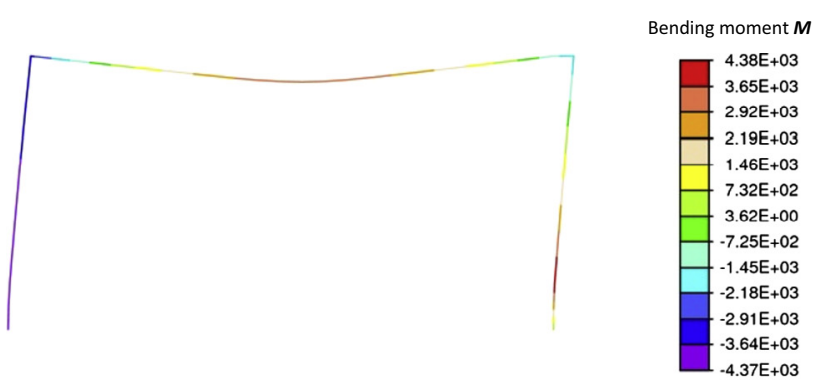

a. Failure in the bending

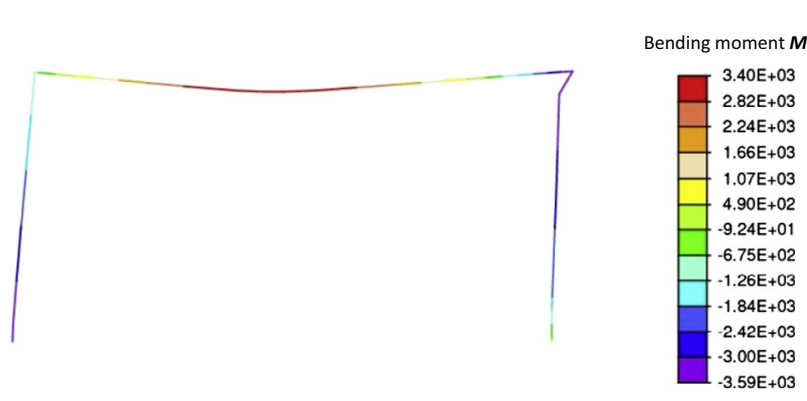

b. Failure in the shearing

Fig. 9. Deformed configuration.

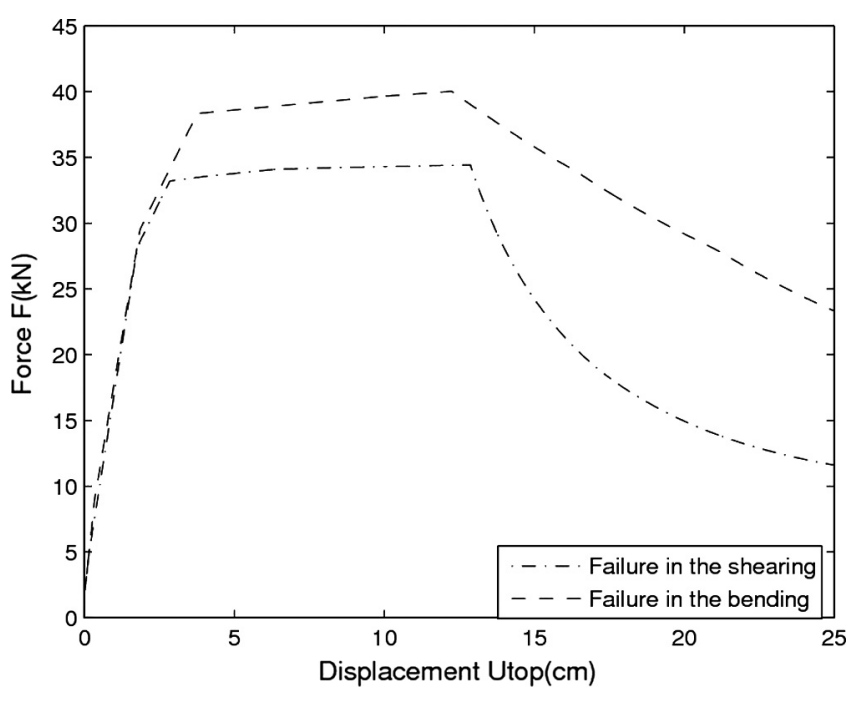

Fig. 10. Response of the frame.

\subsection{Push-over analysis of a simple frame}

In this example, we present ability to capture different failure modes of the frame. We consider a simple steel frame presented in Fig. 8, where the span is $5.0 \mathrm{~m}$ and height is $3.0 \mathrm{~m}$. The mesh is composed of 48 elements where the length of each element is $0.25 \mathrm{~m}$. The material properties of all frame members are equal (Young's modulus: $E=2 \cdot 10^{4} \mathrm{kN} / \mathrm{cm}^{2}$; Hardening modulus: $\mathrm{K}=0.05 \cdot \mathrm{E}$; Moment of inertia: $I=1940 \mathrm{~cm}^{4}$; Area of cross section: $A=28.5 \mathrm{~cm}^{2}$; Yield bending moment: $M_{y}=3100 \mathrm{kN} \mathrm{cm}$; Ultimate bending moment: $M_{u}=3100 \mathrm{kN} \mathrm{cm}$; Yield shear force: $\mathrm{V}_{\mathrm{y}}=355 \mathrm{kN}$; Ultimate shear force: $V_{u}=400 \mathrm{kN}$, Fracture energies: $G_{f, M}=550$ and $G_{f, V}=450$ ), but elements which connect beams and columns are defined according to connection behavior see [6]. Two cases are considered, in the first, connections are defined properly and in the second case connection are defined with very low capacity regard to the shear force $\left(V_{u}=30 \mathrm{kN}\right)$, which can be caused by poor construction during building. This construction error is assumed in the right corner of the steel frame. Deformed configurations of the frame for both cases are presented in Fig. 9. The results of analysis for both cases are presented in Fig. 10, showing a significant reduction in frame limit load that can be brought by construction errors. Fig. 10b shows this reduction of the limit load, caused by construction errors during building.

\section{Conclusions}

The presented geometrically non-linear planar beam model provides the main novelty with its ability to account for both bend- ing and shear failure. The proposed constitutive model contains both coupled plasticity with isotropic hardening and nonlinear law for softening with three different failure mechanisms. The hardening response providing the interaction between bending moment, shear force and axial force can be calibrated against damage of beams or columns in a steel frame. The softening response can be activated to model the failure mode in the connections with different failure mechanisms. Which of mechanisms will be activated depends on interplay and stress redistribution during the limit load analysis.

By using the proposed beam element we can perform ultimate limit analysis of any frame planar steel structure, including the second order effects as well as different failure mechanisms. The geometrically nonlinear analysis allows the ultimate limit analysis with large displacement without any need for correction of the proposed property [1]. This advantage is very important in a steel frame structure because of a large ductility of steel.

The results for all numerical examples illustrate an excellent performance of the proposed beam element.

\section{Acknowledgement}

This work was supported by French Ministry of Foreign Affairs through scholarship given by French Embassy in Sarajevo. This support is gratefully acknowledged.

\section{References}

[1] Dujc J, Bostjan B, Ibrahimbegovic A. Multi-scale computational model for failure analysis of metal frames that includes softening and local buckling. Comput Methods Appl Mech Eng 2010;199:1371-85.

[2] Hill R. The mathematical theory of plasticity. Oxford: Clarendon Press; 1950.

[3] Ibrahimbegovic A. Nonlinear solid mechanics. Springer; 2009.

[4] Ibrahimbegovic A, Frey F. Finite element analysis of linear and non-linear planar deformations of elastic initially curved beam. Int J Numer Meth Eng 1993;36:3239-58.

[5] Ibrahimbegovic A, Frey F. Geometrically non-linear method of incompatible modes in application to finite elasticity with independent rotations. Int J Numer Methods Eng 1993;36:4185-200.

[6] Imamovic I, Ibrahimbegovic A, Knopf-Lenoir C, Mesic E. Plasticity-damage model parameters identification for structural connections. Coupled Syst Mech 2015;4(4):337-64.

[7] Medic S, Dolarevic S, Ibrahimbegovic A. Beam model refinement and reduction. Eng Struct 2013;50:158-69.

[8] Neal BG. Effect of shear and normal forces on the fully plastic moment of a beam of rectangular cross-section. J Mech Eng Sci 1961;23(2).

[9] Nikolic M, Ibrahimbegovic A, Miscevic P. Brittle and ductile failure of rocks: embedded discontinuity approach for representing mode $\mathrm{i}$ and mode ii failure mechanisms. Int J Numer Methods Eng 2015;8(102).

[10] Reissner E. On one-dimensional finite-strain beam theory: the plane problem. Z Angew Math Phys 1972;23.

[11] Simo JC, Hjelmstad KD, Taylor RL. Numerical formulations of elastoviscoplastic response of beams accounting for the effect of shear. Comput Methods Appl Mech Eng 1983;42.

[12] Taylor RL. FEAP - A finite element analysis program. Berkeley; 2008. 